\title{
Maternal and Neonatal Outcomes of Pregnant Women with Clinically Confirmed COVID-19 Admitted at the Philippine General Hospital
}

\author{
Mary Judith Q. Clemente, MD, ${ }^{1}$ Melissa D. Amosco, MD, PhD, ${ }^{1}$ Ma. Bernadette R. Octavio, MD, MSc, ${ }^{1}$ \\ Sybil Lizanne R. Bravo, RPh, MD, MSc ${ }^{1}$ and Esterlita Villanueva-Uy, MD, MSPH ${ }^{2}$ \\ ${ }^{1}$ Department of Obstetrics and Gynecology, Philippine General Hospital, University of the Philippines Manila \\ ${ }^{2}$ Institute of Child Health and Development, National Institutes of Health, University of the Philippines Manila
}

\begin{abstract}
Objectives. The effect of COVID-19 infection in pregnant women and her neonate is not well-understood, with no clear evidence for vertical transmission. This study aims to determine the maternal and neonatal clinical characteristics and the dyad's outcomes among those infected with COVID-19 infection.
\end{abstract}

Methods. An ambispective cross-sectional study involving pregnant women with confirmed COVID-19 infection was conducted at the Philippine General Hospital from April to August 2020. Two hundred nine obstetric patients were included, 14 of whom consented to specimen collection to determine vertical transmission.

Results. The majority of pregnant women with COVID-19 infection and their neonates had good outcomes. Labor, delivery, and the immediate postpartum course were generally uneventful. The all-cause maternal morbidity rate was high at 75.6 per 100 cases during the five-month study period. COVID-19 related morbidities included the development of Guillain-Barré Syndrome. The in-hospital all-cause maternal mortality rate was 1.91 per 100 cases. The causes of maternal death were acute respiratory failure, septic shock, and congenital heart disease (atrial septal defect with Eisenmengerization). The in-hospital, all-cause neonatal mortality rate was 1.04 per 100 neonates of cases. The lone mother and infant deaths were in a postmortem rt-PCR swab negative mother with an rt-PCR swab positive live neonate who eventually succumbed after nine days of life. All 14 dyads with collected specimens that included amniotic fluid, placental tissue, umbilical cord, and neonate nasopharyngeal swab tested negative for SARS-CoV-2 rt-PCR.

Conclusion. The prognosis for COVID-19 infected pregnant patients was generally good, with most of the patients discharged improved. Almost all of the neonates born to COVID-19-infected mothers were stable-term infants. There was no evidence for vertical transmission, as shown by negative rt-PCR results for all the additional specimens obtained.

In general, the prognosis for COVID-19 infected dyads was good. The majority of the mothers were discharged well with their term infants. All possible maternal sources of COVID-19 infection to the neonate tested negative. This study provided no evidence for vertical transmission.

Key Words: COVID-19, maternal outcome, neonatal outcome, pregnant COVID

\section{INTRODUCTION}

Corresponding author: Melissa D. Amosco, MD, PhD Department of Obstetrics and Gynecology Philippine General Hospital University of the Philippines Manila

Taft Avenue, Ermita, Manila 1000, Philippines

Email: mdamosco@up.edu.ph
Since the beginning of the COVID-19 pandemic, many studies have attempted to determine the effects of COVID-19 infection on the pregnant patient and her neonate. Physiologic changes in pregnancy pose a theoretical increased risk for infectious complications. However, the impact of COVID-19 disease on the mother and the neonate is still not fully understood. It is unclear 
if infection in the pregnant woman follows a worse course compared to the nonpregnant patient. It is also unknown whether COVID-19 infection predisposes a pregnant woman to adverse maternal and neonatal outcomes with no clear evidence showing in-utero transmission. Finally, the management of COVID-19 infected pregnant women is experimental at best. With the COVID 19 pandemic still ravaging the Philippines and mass COVID-19 vaccination program waiting for full implementation, obstetricians and pediatricians will continue to deal with maternal and neonatal COVID-19 issues indefinitely. Hence, Philippine data is of utmost importance to elucidate the disease's natural course in the mother-baby dyad. This study aimed to assess pregnant women's clinical characteristics with COVID-19 infection, their clinical course, the maternal and neonatal outcomes, and the likelihood of vertical transmission.

\section{METHODS}

\section{Research design}

This ambispective cross-sectional study collaborated with the Department of Obstetrics and Gynecology and the Department of Pediatrics of the University of the Philippines, Philippine General Hospital (UP-PGH) in Manila with the Declaration of Helsinki 1964. The UP-PGH Ethics Review Board approved the study protocol.

\section{Study population}

The subjects included all consecutive pregnant women at any age of gestation admitted to the institution for medical, obstetric management, or both from April to August 2020 with the following COVID-19 status:

1. COVID-19 infection diagnosed by polymerase chain reaction method;

2. Suspected COVID-19 infection awaiting PCR result but whose clinical presentation is consistent with moderate to severe COVID-19 infection;

3. Negative PCR but whose clinical presentation, other laboratory findings (e.g., chest x-ray), or both are consistent with COVID-19; and

4. Asymptomatic with negative or no PCR but with other laboratory findings consistent with COVID-19 infection.

Informed consent was taken from all eligible patients. Pregnant patients who consented to additional samples for COVID-19 testing were included in the COVID-19 vertical transmission phase of the study.

Excluded were those admitted post-partum or had their delivery outside of the institution.

\section{Conduct of the Study}

Once admitted, all pregnant patients were co-managed by specialists from the Division of Obstetrics and Gynecology Infectious Disease and the Department of Internal
Medicine, when necessary. Interventions were performed as obstetrically indicated.

All infants born to these mothers were admitted to the designated COVID-19 areas for well and unstable infants. They were managed following protocols developed by the Division of Neonatology, Department of Pediatrics, and adapted from the Philippine Pediatric Society's recommendations.

Pertinent maternal and neonatal information were recorded in the data abstraction form. After discharge from the hospital, the clinical course of the mother-baby dyad was routinely followed through telephone, while on quarantine at a designated facility provided by the local government, until a negative PCR test was obtained, or until the $14^{\text {th }}$ day of life for the neonate.

\section{Specimen Collection and Processing}

\section{Maternal samples}

Swab samples from the nasal- or oropharyngeal area were collected per the World Health Organization standard guidelines. ${ }^{1}$ The samples were submitted to the hospital laboratory for real-time reverse-transcription polymerase chain reaction (rt-PCR) assay for COVID-19. Routine laboratory tests for pregnancy/parturition were performed; additional laboratory and radiologic procedures were done when indicated and upon the attending physicians' discretion.

A total of 14 subjects consented to samples to be taken from the mother-baby dyad for further analysis. The maternal samples were taken at the time of delivery under strict sterile conditions included: (1) amniotic fluid (2-3 ml), (2) placental tissue (1 cm block), (3) umbilical cord $(1 \mathrm{~cm}$ block), and (4) breast milk ( $1 \mathrm{ml}$, if available). The amniotic fluid and milk were placed in separate sterile tubes. Blocks of placental tissue and cord were suspended in different tubes with a viral transport medium.

\section{Neonatal samples}

All routine laboratory and radiologic tests recommended for neonates born to COVID-19 mothers were performed. The blood tests included baseline complete blood count with platelet count and blood culture. On the $24^{\text {th }}$ hour of life, blood samples for C-reactive protein, procalcitonin, liver function, and creatinine kinase were obtained. Chest radiographs were done on all infants. Additional tests were done when clinically indicated.

Nasopharyngeal swabs were immediately collected at birth simultaneously with maternal specimens. Gastric aspirate, anal and second nasopharyngeal swabs were obtained on the $24^{\text {th }}$ hour of life. Infants with positive tests had repeat nasopharyngeal and anal swabs on the $7^{\text {th }}$ and $14^{\text {th }}$ day of life. A repeat swab was done on the $21^{\text {st }}$ day of life for neonates with persistent positive results.

All maternal and neonatal samples were stored and sent to the Philippine Genome Center inside proper transport 
containers and collection bags at $2-8^{\circ} \mathrm{C}$. The samples were subjected to nucleic acid amplification tests (NAAT) for the COVID-19 virus using rt-PCR.

\section{Statistical analysis}

All the data were checked, cleaned, encoded, and analyzed using STATA 16.1/1C. The statistical summary was expressed in median and means with corresponding standard deviations for continuous variables. The categorical variables were summarized using frequencies, rates, and proportions.

\section{RESULTS}

\section{Demographic and clinical characteristics}

A total of 209 pregnant women were included in the study. Of these, 197 (94.26\%) patients had positive COVID-19 rt-PCR swabs: 181 were already positive on admission and another 16 tested positive after being admitted. Twelve initially positive patients COVID-19 had negative tests on rt-PCR. Notable is one patient who tested negative for COVID-19 twice using rt-PCR but whose baby tested positive at the NICU.

The patients' mean age was 30.05 years $( \pm 6.13)$, with an age range of 14 to 43 . Most were in the 20-34 years age group $(70.33 \%)$. The majority of the women were multigravidas. A quarter of cases were primigravidas, while the grand multipara represented only one percent of all cases. More than half were unmarried. Eighty percent of the women were from Manila, and the rest were from nearby northern and southern provinces. Only seven (3.35\%) women had a history of travel outside the Philippines. Only fifteen (7.18\%) women reported having a household member with symptoms of COVID-19 infection. Table 1 shows the demographic data of the subjects.

The median age of gestation on admission was 38 weeks, with labor pains as the most common presenting complaint. The majority denied any symptoms related to COVID-19 infection (64.59\%). If COVID-19-related symptoms were reported, cough (19\%) was the most common, followed by fever (10\%) and colds. The most common co-morbidity was hypertension which was present in $22.49 \%$ of cases as gestational or chronic hypertension and pre-eclampsia. Gestational or overt diabetes was present in almost $20 \%$ of cases. Half of the women (49.76\%) did not have any co-morbidity at all.

Baseline physical examination findings revealed that only three patients (1.44\%) presented with body temperatures of $37.5^{\circ} \mathrm{C}$ or higher. Nineteen patients (9.09\%) had elevated blood pressure ( $\geq 140 / 90)$, while two patients (1.0\%) presented with tachypnea (RR >20 CPM). The majority (81.8\%) had no significant chest findings, although auscultation was not possible in 26 patients (12.44\%) due to their protective equipment. Of the 183 patients on whom chest auscultation was possible, only two patients (1.1\%) presented with crackles. Half of the patients were
Table 1. Personal characteristics of pregnant patients with COVID-19 Infection ( $\mathrm{n}=209)$

\begin{tabular}{|c|c|}
\hline Characteristic & n (\%) \\
\hline $\begin{array}{l}\text { Age in years (mean } \pm \text { sem) } \\
\quad \text { Min-max* } \\
\quad \leq 19 \\
20-34 \\
\geq 35\end{array}$ & $\begin{array}{c}30.05 \pm 6.13 \\
14-43 \\
7(3.35) \\
147(70.33) \\
55(26.32)\end{array}$ \\
\hline $\begin{array}{l}\text { Gravidity (med, IQR) } \\
\text { Gravidity (\%) } \\
\quad \text { G1 } \\
\text { G2-5 } \\
\text { > G6 }\end{array}$ & $\begin{array}{c}2(2) \\
60(28.71) \\
145(69.38) \\
4(1.91)\end{array}$ \\
\hline 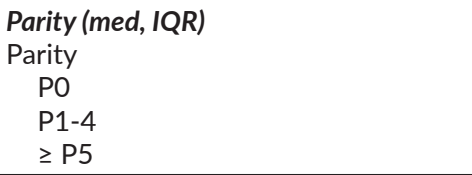 & $\begin{array}{c}1(2) \\
80(38.28) \\
127(60.76) \\
2(0.96)\end{array}$ \\
\hline $\begin{array}{l}\text { AOG in weeks (med, IQR) } \\
\text { Weight in kilograms (med, IQR) } \\
\text { Height in centimeters (med, IQR) } \\
\text { BMI in } \mathrm{kg} / \mathrm{m}^{2} \text { (med, IQR) }\end{array}$ & $\begin{array}{c}38.43(1.86) \\
67(16) \\
155(8) \\
28.16(5.8) \\
\end{array}$ \\
\hline $\begin{array}{l}\text { Civil status } \\
\text { Single } \\
\text { Ever married } \\
\end{array}$ & $\begin{array}{c}116(55.50) \\
93(44.50)\end{array}$ \\
\hline $\begin{array}{l}\text { Residence } \\
\text { Manila } \\
\text { Luzon provinces north of Manila } \\
\text { Luzon provinces south of Manila } \\
\text { Mindanao }\end{array}$ & $\begin{array}{c}167(79.90) \\
5(2.39) \\
30(14.35) \\
2(0.96)\end{array}$ \\
\hline $\begin{array}{l}\text { Educational Attainment } \\
\text { None } \\
\text { Grade school } \\
\text { High school } \\
\text { College } \\
\text { Vocational }\end{array}$ & $\begin{array}{c}3(1.44) \\
11(5.26) \\
67(32.06) \\
113(54.07) \\
2(0.96)\end{array}$ \\
\hline $\begin{array}{l}\text { Gross family monthly income (Php) } \\
\quad<10,000 \\
\quad 10,000-50,000 \\
>100,000 \\
\text { Missing data }\end{array}$ & $\begin{array}{c}18(8.61) \\
55(26.32) \\
1(0.48) \\
135(64.59)\end{array}$ \\
\hline $\begin{array}{l}\text { Work } \\
\text { Employed } \\
\text { Unemployed } \\
\text { Student } \\
\text { OFW }\end{array}$ & $\begin{array}{c}93(44.50) \\
80(38.28) \\
4(1.91) \\
3(1.44)\end{array}$ \\
\hline $\begin{array}{l}\text { Travel history } \\
\text { Travel history location, Middle East }\end{array}$ & $\begin{array}{l}7(3.35) \\
4(57.14)\end{array}$ \\
\hline $\begin{array}{l}\text { Household member with symptoms } \\
\text { Spouse } \\
\text { Son/daughter } \\
\text { Extended family } \\
\text { Helper }\end{array}$ & $\begin{array}{l}15(7.18) \\
8(53.33) \\
2(13.33) \\
8(3.83) \\
1(0.48)\end{array}$ \\
\hline
\end{tabular}

AOG, Age of gestation; sem, standard error of the mean; BMI, body mass index; med, median; iqr, interquartile range

in early labor with the median cervical dilatation of two centimeters or less. The majority of the fetuses (85.58\%) were in cephalic presentation.

The majority of patients had normal complete blood count results: median hemoglobin of 12.4 grams/dL, median 
Table 2. Pregnancy outcomes and manner of delivery $(n=209)$

\begin{tabular}{|c|c|c|c|c|}
\hline Pregnancy Outcome & Number $(n=210)$ & Manner of Delivery & Number ( $n=196)$ & Total n (\%) \\
\hline \multirow[t]{6}{*}{ Livebirths (includes one twin delivery) } & 192 & & 191 & \\
\hline & & SVD & 64 & \\
\hline & & Assisted - Forceps & 4 & \\
\hline & & Assisted - Vacuum & 3 & $76(37.17 \%)$ \\
\hline & & Abdominal - LSCS & 111 & \\
\hline & & Abdominal - Classical & 9 & $120(62.83 \%)$ \\
\hline Fetal death-in-utero & 2 & SVD & & \\
\hline Late abortion & 3 & Spontaneous expulsion of abortus & & \\
\hline Ectopic pregnancy & 2 & Salpingectomy & & \\
\hline Undelivered & 11 & & & \\
\hline
\end{tabular}

SVD, Spontaneous vaginal delivery; LSCS, low segment cesarean section

white blood cell count of $11.4 \times 10^{9}$ cells per liter, and median platelet of $279 \times 10^{9} / \mathrm{L}$. Chest radiograph results from 156 patients (74.64\%) revealed that only a third of patients (58) had findings consistent with pneumonia. Forty-eight patients had no significant chest findings on chest radiographs.

For the patients' COVID-19-related course, 142 patients $(72.08 \%)$ were diagnosed with pneumonia based on clinical, radiographic, or both findings. Five patients (3.52\%) needed mechanical ventilation, with two progressed to adult respiratory distress syndrome. Two patients developed cytokine storms. One patient developed GuillainBarré Syndrome. Two had renal insufficiency, with one required hemodialysis.

\section{Conduct of Labor and Delivery in the COVID-19 Ward Setting}

Almost all (99.04\%) of the patients wore at least a face mask during labor, delivery, and postpartum. Only one patient was placed under negative-pressure isolation. The majority of the patients $(174,83.25 \%)$ underwent labor trials with a median duration of 4.9 hours. Nineteen patients (9.09\%) underwent control of preterm labor with either nifedipine or isoxsuprine. In eight patients, management of preterm labor was unsuccessful.

Almost half of the patients $(97,46.41 \%)$ were given both uterotonics, oxytocin, and carbetocin. Cefazolin was the most frequently administered antibiotic prophylaxis for cesarean section. Anticoagulants were administered in nine (9) patients in the form of heparin or enoxaparin.

The median estimated blood loss during delivery was $500 \mathrm{ml}$. Six (6) patients received blood transfusion: three (3) for correction of preexisting anemia and three (3) for volume replacement during cesarean section and postpartum hemorrhage. The average number of blood transfused was 2.5 units.

The practice of essential intrapartum and newborn care (EINC) was greatly affected. Although the majority (98.51\%) of the babies were immediately dried after delivery, early skin-to-skin contact and non-separation of the mother and baby were not carried out in almost all dyads. Breastfeeding was likewise withheld for the majority of babies (99\%). One
Table 3. Indications for Cesarean Section

\begin{tabular}{lcc} 
Indication & $\begin{array}{c}\text { Frequency } \\
\mathbf{n = 1 2 0}\end{array}$ & $\begin{array}{c}\text { Relative } \\
\text { Frequency }\end{array}$ \\
Scarred uterus & 61 & 50.83 \\
Non-reassuring fetal heart rate pattern & 15 & 12.50 \\
Dystocia & 14 & 11.67 \\
Relative maternal indication & 10 & 8.33 \\
Malpresentation & 6 & 5.00 \\
Per COVID-19 protocol & 6 & 5.00 \\
Multifetal pregnancy & 2 & 1.67 \\
Placenta previa & 1 & 0.83 \\
Others & 5 & 4.17 \\
\hline
\end{tabular}

hundred ninety-two neonates were directly admitted to the neonatal intensive care unit as per COVID-19 protocol.

\section{Pregnancy-related maternal outcomes}

Table 2 shows the pregnancy outcomes and manner of delivery. There were 196 (93.88\%) deliveries and two salpingectomies for ectopic pregnancy during the index admission. Eleven were sent home undelivered after control of preterm labor. Almost two-thirds of patients were delivered abdominally. The majority of the cesarean section indications were obstetric, and the most common of which was for scarred uteri. Only six patients (5.04\%) underwent cesarean section per COVID-19 protocol. Table 3 shows the indications for abdominal delivery.

There were five (5) fetal wastages: two (2) third-trimester fetal deaths in-utero and three (3) late abortions. Table 4 shows the clinical characteristics and pregnancy outcomes of this subset of patients. The majority were in their advanced age and had co-morbidities.

\section{Maternal Morbidity and Mortality}

The overall maternal morbidity rate was high at $75.6 \%$ (158/209) during the five-month study period. In a subset of patients who were confirmed positive on rt-PCR, the overall maternal morbidity rate is $78.17 \%(154 / 197)$. These morbidities included anemia and postpartum hemorrhage necessitating blood transfusion, uterine atony, puerperal fever, surgical site infection, pneumonia, cytokine storm, and renal insufficiency. 
Table 4. Clinical characteristics and outcome of pregnancy with late abortion and fetal death in-utero

\begin{tabular}{|c|c|c|c|}
\hline $\begin{array}{l}\text { Maternal age, in } \\
\text { years, Gravidity } \\
\text { and Parity (G/P) }\end{array}$ & $\begin{array}{c}\text { Amenorrhea } \\
\text { (weeks) }\end{array}$ & $\begin{array}{c}\text { Maternal } \\
\text { Co-morbidity }\end{array}$ & $\begin{array}{l}\text { Fetal/neonatal } \\
\text { birth weight } \\
\text { (grams) }\end{array}$ \\
\hline \multicolumn{4}{|l|}{ Late abortion } \\
\hline $40, \mathrm{G} 1$ & 28 & $\mathrm{CH}$ & 240 \\
\hline 38, G4P2 & 31 & GC, PPC & 245 \\
\hline 43, G3P1 & 18 & none & 200 \\
\hline \multicolumn{4}{|l|}{ Fetal death in-utero } \\
\hline 37, G3P2 & 34 & PE, GDM & 1900 \\
\hline 30, G2P1 & 38 & none & 2730 \\
\hline
\end{tabular}

$\mathrm{CH}$, Chronic hypertension; GC, Gravidocardia; PPC, Post-partum cardiomyopathy; PE, Preeclampsia; GDM, Gestational diabetes mellitus

Table 5. Frequency distribution of maternal morbidity and mortality $(n=209)$

\begin{tabular}{lrr} 
& Frequency & $\begin{array}{c}\text { Relative } \\
\text { Frequency }\end{array}$ \\
\hline Morbidity ( $\boldsymbol{n}=158)$ & & \\
COVID-19-related & & \\
$\quad$ Pneumonia & 142 & 68.0 \\
$\quad$ Mechanical ventilatory support & $(5)$ & $(2.39)$ \\
$\quad$ Acute Respiratory Distress Syndrome & $(2)$ & $(0.96)$ \\
Cytokine storm & 2 & 0.96 \\
$\quad$ Guillain-Barré Syndrome & 1 & 0.48 \\
$\quad$ Renal insufficiency & 2 & 0.86 \\
$\quad$ Hemodialysis & $(1)$ & $(0.48)$ \\
Direct Obstetrical & 3 & 1.44 \\
$\quad$ Puerperal fever & 1 & 0.48 \\
$\quad$ Surgical site infection & 1 & 0.48 \\
$\quad$ Uterine Atony & 6 & 2.87 \\
$\quad$ Need for blood transfusion & & \\
\hline Mortality ( $n=4)$ & 2 & 0.96 \\
Acute respiratory failure & & \\
$\quad$ Pulmonary embolism & & \\
$\quad$ Severe COVID-19 pneumonia & 1 & 0.48 \\
Septic shock & 1 & 0.48 \\
Congenital heart disease (atrial septal & & \\
$\quad$ defect with Eisenmengerization) & & \\
$\quad$ and HELLP syndrome & &
\end{tabular}

Notable was a case of Guillain-Barré Syndrome (GBS) in a secundigravida at 22 weeks gestational age with a three-week history of upper respiratory infection and generalized body weakness. Based on the presenting symptoms and the findings on cerebrospinal fluid analysis, the co-managing Neurology service administered a fiveday course of intravenous immunoglobulin that afforded marked improvement of neurologic symptoms. Her repeat rt-PCR swab on day 14 of illness was negative. At term, the patient delivered to a healthy baby at another hospital.

The in-hospital, all-cause mortality rate was 1.91 per 100 cases. In a subset of confirmed positive on rt-PCR patients, the in-hospital all-cause mortality rate was 1.52 per 100 COVID-19-positive cases. There were four deaths with the following causes: pulmonary embolism during the second stage of labor, severe COVID-19 pneumonia, septic shock with diabetic ketoacidosis, and atrial septal defect, and Eisenmengerization with HELLP syndrome. Interestingly, the latter patient with congenital heart disease was negative for COVID-19 twice using rt-PCR but whose baby tested positive at the neonatal intensive care unit. Table 5 shows the frequency distribution of the causes of maternal morbidity and mortality.

\section{Outcomes of Neonates born to COVID-19 confirmed mothers}

One hundred ninety (191) COVID-19 confirmed mothers delivered 192 live-born infants, all singletons except for one set of twins. The mean pediatric aging was 38.4 weeks $( \pm 1.64)$, ranging from 29 to 41 weeks. The mean birth weight was $2,927.96$ gram $( \pm 496.31 ; 616-4,630)$. Apgar scores were $8.6 \pm 1.36$ and $8.8 \pm 0.79$ at 1 and 5 mins, respectively (Table 6).

After birth, all the infants were immediately dried except one infant, who was cyanotic and limp. Immediate cord clamping and immediate separation from the mother were performed in all the infants. Fourteen neonates (7.30\%) required ventilatory support: six (6) needed free-flow oxygen, two (2) had bag-mask ventilation, and six (6) were intubated. Of the 192 neonates, 13 (6.77\%) were admitted to the Level III NICU, while the rest were observed in the Intermediate Care Unit (Level II) and the Well baby COVID-19 ward (Level 1).

Some neonates who were rt-PCR negative had clinical manifestations related to COVID-19 infections such as tachycardia (12), tachypnea (2), hypotension (2), and vomiting (1). However, none of the neonates exhibited abnormal body temperature or lethargy.

Only two neonates (1.04\%) tested positive for SARSCOV-2 virus by rt-PCR.

Neonate 1 was a preterm 31-weeker, growth-restricted with a birth weight of 930 grams. Endotracheal aspirate on the $24^{\text {th }}$ hour of life was positive for SARS-COV-2 by rtPCR. He had a stormy course characterized by respiratory distress syndrome, patent ductus arteriosus, necrotizing enterocolitis, ileus, anemia, and thrombocytopenia. Cultures showed late-onset sepsis. He succumbed after nine days, and the cause of death was septic shock with disseminated intravascular coagulation.

Neonate 2 was a term 37 -weeker with a birth weight of 2,775 grams with good APGAR scores but tested positive for SARS-COV-2 from his nasopharyngeal swab. Immediate drying and early cord clamping were performed. The infant was not immediately separated from the mother. The neonate remained stable except for two episodes of vomiting on the first day of life. He was discharged after three days. No repeat swab was obtained.

The most commonly observed neonatal morbidities were respiratory: neonatal pneumonia (5), transient tachypnea of the newborn (13), respiratory distress syndrome (3), and pneumothorax (1). Other morbidities included: hypoxicischemic encephalopathy (1) and seizure (1). Two (2) neonates 
Table 6. Clinical characteristics of neonates born to COVID19 confirmed infected mothers $(n=192)$

\begin{tabular}{lcc|}
\multicolumn{1}{c}{ Criteria } & Average & Range \\
\hline Pediatric Aging (weeks) & $38.39 \pm 1.64$ & $29-41$ \\
Birth-weight (g) & $2,927.96 \pm 496.31$ & $616-4630$ \\
Head circumference (cm) & $33.60 \pm 2.74$ & $22-41$ \\
Length *cm) & $47.85 \pm 6.08$ & $31-56$ \\
Apgar Score at 1 min & $8.6 \pm 1.36$ & \\
Apgar Score at 5 mins & $8.85 \pm 0.79$ & \\
\multicolumn{1}{c}{ Outcomes } & Number & Percentage \\
\hline Livebirths & 192 & \\
Singletons & 191 & 99.47 \\
Set of twins & 1 & 0.53 \\
Term & 182 & 94.79 \\
$\quad$ Preterm & 10 & 5.20 \\
SARS COV-2 positive test & 2 & 1.04 \\
$\quad$ Nasopharyngeal swab & 1 & \\
Endotracheal aspirate & 1 & 98.96 \\
SARS COV-2 negative test & 190 & \\
\hline
\end{tabular}

NICU, Neonatal intensive care unit; NPS, nasopharyngeal swab; ET, endotracheal

had congenital heart disease and one (1) with lumbosacral meningocele.

There were two neonatal deaths. The in-hospital, allcause neonatal mortality rate was 1.04 per 100 neonates of COVID-19 maternal cases. One was a term infant (38 weeks), birth-weight of 3,251 grams, severely asphyxiated (AS 0,1 , and 3 at 1,5 , and 10 minutes, respectively). The neonate succumbed after 30 days at the NICU with the diagnosis of hypoxic-ischemic encephalopathy. The other mortality was the preterm growth-restricted neonate mentioned earlier.

\section{Maternal-neonatal dyad with additional samples subjected to rt-PCR}

The demographic and clinical characteristics of the 14 women who consented to the collection of maternal and neonatal samples are outlined in Table 7. All 14 patients had a positive rt-PCR test before admission. One had pneumonia. All patients delivered abdominally, nine for scarred uteri and five for obstetric indications.

All samples collected from the dyad (amniotic fluid, placenta, umbilical cord, breast milk, neonatal gastric aspirate, anal swab, and nasopharyngeal swab at birth) tested negative for SARS-CoV-2 using the rt-PCR technique.

\section{DISCUSSION}

Many questions remain unanswered as far as COVID-19 infection in pregnancy is concerned. It is unknown whether pregnancy increases the risk of acquiring the disease or if the condition leads to poorer maternal and fetal outcomes. Vertical transmission is likewise unclear. Pregnancy itself may limit the use of certain drugs or interventions that are otherwise available for use in the nonpregnant patient. There is no consensus as to the optimal timing and mode of
Table 7. Demographic and clinical characteristics of maternal and fetal dyads with submitted samples for analysis ( $n=14$ )

\begin{tabular}{|c|c|c|}
\hline Criteria & Average & Range \\
\hline \multicolumn{3}{|l|}{ Maternal } \\
\hline Age (years) & $31 \pm 6.4$ & $17-42$ \\
\hline BMI & $29.4 \pm 2.9$ & $26.0-34.2$ \\
\hline AOG (weeks, days) & $38 w 5 d \pm 1 w 1 d$ & $36 w 6 d-41 w 4 d$ \\
\hline Gravidity & $2.2 \pm 0.97$ & $1-4$ \\
\hline \multicolumn{3}{|l|}{ Neonatal } \\
\hline \multirow[t]{2}{*}{ Birth-weight } & $2840.7 \pm 347.9$ & \\
\hline & $\begin{array}{c}\text { Percentage } \\
\mathrm{n}=14(\%)\end{array}$ & \\
\hline \multicolumn{3}{|l|}{ Reason for admission: } \\
\hline Obstetrics (labor pains) & $14(100)$ & \\
\hline \multicolumn{3}{|l|}{ Manner of delivery } \\
\hline Repeat CS & $9(64.3)$ & \\
\hline CS for malpresentation & $1(7.1)$ & \\
\hline CS for other obstetric indication & $4(28.6)$ & \\
\hline \multicolumn{3}{|l|}{ Maternal co-morbidities } \\
\hline Hypertension/preeclampsia & $4(28.6)$ & \\
\hline Gestational diabetes mellitus & $2(14.2)$ & \\
\hline \multicolumn{3}{|l|}{ COVID-19-related symptoms } \\
\hline Cough/colds & $1(7.1)$ & \\
\hline No related symptoms & $13(92.9)$ & \\
\hline rt-PCR positive on admission & $14(100)$ & \\
\hline Pneumonia positive & $1(7.1)$ & \\
\hline
\end{tabular}

BMI, body mass index; AOG, Age of gestation; CS, Cesarean section; $r t-P C R$, reverse-transcription-polymerase chain reaction

delivery for the pregnant COVID-19 patient. The same holds for postpartum management and neonatal interventions. ${ }^{2}$

Being the first national COVID-19-referral center, the PGH served as a catchment for infected pregnant patients from all over the metropolis, neighboring provinces, and as far down south as Mindanao. Aside from the COVID-19 infection, most of the patients admitted did not have comorbid conditions at term and were in labor.

Except for the donning of personal protective equipment, as mandated by hospital protocols, labor and delivery management generally followed existing standards. The cesarean section rate for this cohort was high at $62.83 \%$. The majority of the indications were obstetric. Only 5\% of patients underwent cesarean section based on the initial COVID-19 protocol. There was great uncertainty about the risk of labor and vaginal delivery for the parturient and the neonate during the first half of 2020. Expediting delivery in a controlled setting, such as in cesarean section, was deemed safer for the dyad and the delivery staff. As new evidence emerged, vaginal delivery is generally safe and well-tolerated for the stable parturient, and COVID-19 infection was not an indication for abdominal delivery.

Similar to previous studies, ${ }^{3,4}$ the two most common COVID-19-related symptoms were cough and fever. Community transmission was the prevalent mode of acquiring the infection as only four patients had a travel history outside the country. Also, 15 patients reported having a household member or close contact with COVID-19-related symptoms 
underscoring the importance of compliance to COVID-19 public health safety protocols in and out of one's residence.

Pregnant women are believed to be vulnerable to many infections due to the immune response's altered physiology. Moreover, lung function physiologic changes such as increased oxygen demand, decreased functional residual capacity, and edema of the respiratory mucosa contribute to a greater risk of hypoxia commonly seen in COVID-19 infection. Recent data have shown that pregnancy is an independent risk factor for adverse COVID-19 outcomes, such as higher odds of pneumonia, ICU admission, and death compared to their nonpregnant counterpart. ${ }^{5}$ Pneumonia was present in almost three-quarters of this pregnant cohort; only five (3.4\%) required mechanical ventilation. The prevalence of preterm labor and preterm delivery for this cohort were $9.1 \%$ and $3.83 \%$, respectively. These figures were far lower than the annual preterm live births at the $\mathrm{PGH}$, which ranged from $22-25 \%{ }^{6}$

Interestingly, a case of Guillain-Barré Syndrome (GBS) with COVID-19 infection was observed in this study. Central or peripheral nervous system involvement is shared with earlier coronavirus infections, SARS and MERS. Immune activation in response to antigen causes injury to the nerve root and peripheral nerves. The damage stems from the structural similarity of the antigens to the myelin and axons. ${ }^{7}$ In most cases, the onset of neurologic symptoms from COVID-19 infection is approximately between a week to 4 weeks like this index case. In some instances, GBS symptoms manifest concurrently with COVID-19 diagnosis giving a "parainfectious" pattern. ${ }^{8}$ The most common symptom is muscle weakness, as seen in the patient, usually beginning in the limbs. Other types may present as flaccid paralysis, demyelinating polyneuropathy, and axonal variants. ${ }^{9}$ Severe paralysis may ensue; that is why it is crucial to recognize GBS symptoms related to a diagnosis of SARS-CoV-2 infection.

The four mortalities account for $1.9 \%$ of the cases in the study. Except for the patient with pulmonary embolism, which is a direct maternal death, the three remaining cases had co-morbidities (sepsis, diabetes mellitus, and Eisenmengerization) that made them at risk for fatal COVID-19 infection.

\section{Neonatal outcomes and transmission of infection}

The majority of the infants in this study were born term, exhibited good APGAR scores, and with birth weights appropriate for gestational age. The prevalence of transient tachypnea in the newborn (TTN) is 6.8\% and defined as rapid breathing ( $>60$ breaths $/ \mathrm{min}$ ) in the first two hours of life with signs of respiratory distress. This rate is higher than the background risk of $1-2 \%$, but within the acceptable range considering that TTN could run as high as $13 \%$ among late preterm infants and those delivered by elective cesarean section. ${ }^{10}$

For all 14 dyads enrolled, albeit a limited number, to test for vertical transmission, all collected specimens that included amniotic fluid, placental tissue, umbilical cord, and neonate nasopharyngeal swab tested negative for rt-PCR SARS-CoV-2. This finding is similar to the results from another study that collected the same samples. ${ }^{3}$ Vertical transmission has been reported to be unlikely, although a small case series have shown the possibility of transmission, albeit very low. ${ }^{11} \mathrm{~A}$ longer time interval between birth to the diagnosis of neonatal COVID-19 infection may indicate horizontal rather than vertical transmission. ${ }^{12} \mathrm{~A}$ systematic review and meta-analysis using samples similar to this study showed the possibility of vertical transmission in a minority of cases..$^{13}$ Hence, larger cohorts are needed to be able to ascertain vertical transmission.

Two neonates, outside of the 14 dyads, tested positive for COVID-19 infection. The prevalence rate of COVID-19 infection in this cohort of infants is $1.04 \%$. Both infants presented with gastrointestinal symptoms, which have been observed in $8.6 \%$ of infected neonates. Majority presented with respiratory distress (41.4\%), fever (15.5\%), lethargy (5.4\%), and poor feeding (5.2\%). However, almost a quarter of COVID-19 infected infants (22.4\%) were asymptomatic. ${ }^{14}$

\section{rt-PCR-negative mother with rt-PCR-positive neonate}

There is a lack of data regarding situations wherein the mother tested negative, but the neonate tested positive after delivery, both using rt-PCR for COVID-19. Considering that rt-PCR was done twice on the mother and the results were both negative, the plausible explanations could be any of the following: the patient is not currently infected by this virus or is too early or too late for the infection to detect the replicating virus. ${ }^{15}$ The false-negative PCR test results can be due to individual variability in the amount of viral shedding ${ }^{16}$ or may represent clearance of the infection. ${ }^{17}$ A false-negative result can happen with poor sample techniques (the virus is not present at the site where the sample was taken from, poor quality of the sample taken), incorrect timing of specimen collection, and incorrect sample handling and storage.

\section{CONCLUSION}

The physiologic changes in pregnancy may place a pregnant woman at a theoretically increased risk for infections. The findings of this study showed that the general prognosis of COVID-19-infected pregnant patients is good. Most presented with mild symptoms and were discharged improved. The course of labor, delivery, and the postpartum period was also generally uneventful for most patients. Almost all of the neonates born to COVID-19-infected mothers were stable-term infants. There were two COVID-19-infected infants, one of whom died due to extreme prematurity and late-onset sepsis. Both infants presented with gastrointestinal symptoms like vomiting. Vertical transmission cannot be ascertained definitely since one COVID-19 
infected infant had a COVID-19 negative mother. The other COVID-19-infected infant had a COVID-19 confirmed mother by rt-PCR of the nasopharyngeal swab, but no other maternal specimens were collected. As shown by negative rt-PCR results for all the additional specimens obtained, we found no possible evidence for vertical transmission.

\section{Acknowledgment}

This study was supported by a faculty grant from the University of the Philippines-Philippine General Hospital, Expanded Hospital Research Office (EHRO) to collect, analyze, and interpret data and write the manuscript.

\section{Statement of Authorship}

Mary Judith Clemente, MD, and Melissa D. Amosco, $\mathrm{MD}, \mathrm{PhD}$, contributed equally to this work.

All authors participated in the data collection and analysis and approved the final version submitted.

\section{Author Disclosure}

All authors declared no conflicts of interest.

\section{Funding Source}

This paper was funded by the University of the Philippines-Philippine General Hospital, Expanded Hospital Research Office (EHRO).

\section{REFERENCES}

1. World Health Organization Interim guidance: Laboratory testing for 2019 novel coronavirus (2019-nCoV) in suspected human cases [Internet]. 2020 [cited February 2021]. Available from: https:// www.who.int/publicationsdetail/laboratory-testing-for-2019-novelcoronavirus-in-suspected-human-cases-20200117

2. Gujski M, Humeniuk E, Bojar I. Current State of Knowledge About SARS-CoV-2 and COVID-19 Disease in Pregnant Women. Med Sci Monit. 2020 May; 26:e924725.

3. Chen H, Guo J, Wang C, Luo F, Yu X, Zhang W, et al. Clinical characteristics and intrauterine vertical transmission potential of COVID-19 infection in nine pregnant women: a retrospective review of medical records. The Lancet. 2020; 395:809-15.
4. Capobianco G, Saderi L, Aliberti S, Mondoni M, Piana A, Dessole F, et al. COVID-19 in pregnant women: A systematic review and metaanalysis. Eur J Obstet Gynecol Reprod Biol. 2020 Sep; 252:543-58.

5. Martinez-Portilla RJ, Sotiriadis A, Chatzakis C, Torres-Torres J, Espino Y Sosa S, Sandoval-Mandujano K, et al. Pregnant women with SARS-CoV-2 infection are at higher risk of death and pneumonia: propensity score matched analysis of a nationwide prospective cohort (COV19Mx). Ultrasound Obstet Gynecol. 2021 Feb; 57(2):224-31.

6. Guinto VL, Domingo M. Epidemiology. CPG Preterm Labor 2015.

7. Yuki N, Hartung HP. Guillain-Barré syndrome. New Engl J Med. 2012 Jun; 366(24):2294-304.

8. Zhao H, Shen D, Zhou H, Liu J, Chen S. Guillain-Barré syndrome associated with SARS-CoV-2 infection: causality or coincidence? Lancet Neurol. 2020 May; 19(5):383-4.

9. Rahimi K. Guillain-Barre syndrome during COVID-19 pandemic: an overview of the reports. Neurol Sci. 2020 Nov; 41(11):3149-56.

10. Moresco L, Romantsik O, Calevo MG, Bruschettini M. Non-invasive respiratory support for the management of transient tachypnea of the newborn. Cochrane Database Syst Rev. 2020 Apr; 4(4):CD013231.

11. Antonakou A. The latest update on the effects of COVID-19 infection in pregnancy. Eur J Midwifery. 2020 Apr; 4:12.

12. Solís-García G, Gutiérrez-Vélez A, Pescador Chamorro I, ZamoraFlores E, Vigil-Vázquez S, Rodríguez-Corrales E, et al. Epidemiology, management and risk of SARS-CoV-2 transmission in a cohort of newborns born to mothers diagnosed with COVID-19 infection. An Pediatr (Eng1 Ed). 2021 Jan. doi: 10.1016/j.anpede.2020.12.006. Epub ahead of print.

13. Kotlyar AM, Grechukhina O, Chen A, Popkhadze S, Grimshaw A, Tal O, Taylor HS, Tal R. Vertical transmission of coronavirus disease 2019: a systematic review and meta-analysis. Am J Obstet Gynecol. 2021 Jan;224(1):35-53.e3.

14. Dhir SK, Kumar J, Meena J, Kumar P. Clinical Features and Outcome of SARS-CoV-2 Infection in Neonates: A Systematic Review. J Trop Pediatr. 2020 Aug: fmaa059.

15. Sanduzzi A, Zamparelli SS. Nasopharyngeal and Oropharyngeal Swabs, And/Or Serology for SARS COVID-19: What Are We Looking For? Int J Environ Res Public Health. 2020 May; 17(9):3289.

16. He X, Lau EHY, Wu P, Deng X, Wang J, Hao X, et al. Temporal dynamics in viral shedding and transmissibility of COVID-19. Nat Med. 2020 Sep; 26(9):1491-3.

17. Woloshin S, Patel N, Kesselheim AS. False Negative Tests for SARSCoV-2 Infection - Challenges and Implications. N Engl J Med. 2020 Aug; 383(6):e38. 